\title{
Geometrical scaling for identified particles
}

\author{
Michal Praszalowicz ${ }^{1}$ \\ M. Smoluchowski Institute of Physics, Jagellonian University, \\ Reymonta 4, 30-059 Krakow, Poland
}

\begin{abstract}
We show that recently measured transverse momentum spectra of identified particles exhibit geometrical scaling (GS) in scaling variable $\tau_{\tilde{m}_{\mathrm{T}}}=$ $\left(\tilde{m}_{\mathrm{T}} / Q_{0}\right)^{2}\left(\tilde{m}_{\mathrm{T}} / W\right)^{\lambda}$ where $\tilde{m}_{\mathrm{T}}=\sqrt{m^{2}+p_{\mathrm{T}}^{2}}-m$. We explore consequences of GS and show that both mid rapidity multiplicity and mean transverse momenta grow as powers of scattering energy. Furthermore, assuming Tsallislike parametrization of the spectra we calculate the coefficients of this growth. We also show that Tsallis temperature is related to the average saturation scale.
\end{abstract}

\section{Introduction}

Geometrical scaling (GS) [1] has been observed both in Deep Inelastic Scattering (DIS) at HERA [2, 3] and in particle production in hadronic collisions [4, 5, 6]. It is an immediate consequence of the existence of an intermediate energy scale called saturation scale, denoted hereafter as $Q_{\mathrm{s}}$. Saturation [7, 8] (for a review see [9, 10]) appears due to the nonlinearities of parton evolution at small Bjorken $x$. General form of this evolution is given by the so-called JIMWLK equations [11] which in the large $N_{\mathrm{c}}$ limit reduce to the Balitsky-Kovchegov equation [12]. These equations have traveling wave solutions which explicitly exhibit GS [13]. An effective theory relevant for the small Bjorken $x$ region is so called Color Glass Condensate (CGC) [14]. For the purpose of present work the details of the saturation are not of primary importance; it is the very existence of the saturation scale which plays the crucial role.

\footnotetext{
${ }^{1}$ e-mail: michal@if .uj.edu.pl
} 
For processes with an external scale $\left(Q^{2}\right.$ in DIS or $p_{\mathrm{T}}^{2}$ of an observed particle produced in hadron-hadron scattering) larger than a typical nonperturbative energy scale $\Lambda^{2}$ being of the order of a couple of hundreds $\mathrm{MeV}$, and smaller than, say, $10 \mathrm{GeV}$ where perturbative QCD can be applied, observables like photon-proton cross-section or charged particle multiplicity depend only upon the ratio of this scale to $Q_{\mathrm{s}}^{2}$ called scaling variable $\tau$. This property is referred to as geometrical scaling [1. However, in a situation where two (or more) external energy scales are present, there exist more than one such ratios, what implies violation or at least modification of GS. Indeed, as we have shown in Ref. [15], particle production in forward rapidity region provides a bona fide example of GS violation. Another possible case of interest are spectra of identified particles where particle masses provide yet another external energy scale that might lead to GS violation [16]. It is the purpose of the present paper to see whether this is really the case.

It is well known that particle spectra at low and medium transverse momenta can be described by thermal distributions in transverse mass $m_{\mathrm{T}}=$ $\sqrt{p_{\mathrm{T}}^{2}+m^{2}}$ with "temperature" $T$ which is a function of the scattering energy [17. One may therefore hope that in some limited range geometrical scaling can be still present with pertinent scaling variable being $m_{\mathrm{T}}^{2} / Q_{\mathrm{s}}^{2}$. It is also known that more accurate fits are obtained by means of Tsallis-like parametrization [18] where particle multiplicity distribution takes the following form (see e.g. [19]):

$$
\frac{1}{p_{\mathrm{T}}} \frac{d^{2} N}{d y d p_{\mathrm{T}}}=C \frac{d N}{d y}\left[1+\frac{m_{\mathrm{T}}-m}{n T}\right]^{-n}
$$

with

$$
C=\frac{(n-1)(n-2)}{n T(n T+(n-2) m)} .
$$

Coefficient $C$ in Eq. (2) ensures proper normalization of (1). Here $n$ and $T$ are free fit parameters that depend on particle species. In the limit $n \rightarrow \infty$ distribution (1) tends to the exponent $\exp \left(-m_{\mathrm{T}} / T\right)$, i.e. to the thermal distribution mentioned above. Formula (1) suggests yet another possibility of scaling variable, namely $\tilde{m}_{\mathrm{T}}^{2} / Q_{\mathrm{s}}^{2}$ where

$$
\tilde{m}_{\mathrm{T}}=m_{\mathrm{T}}-m=\sqrt{p_{\mathrm{T}}^{2}+m^{2}}-m \text {. }
$$

Subtracting $m$ from $m_{\mathrm{T}}$ in (3) contributes in the large $n$ limit to an overall factor and does not influence the functional dependence of thermal distribution. For finite $n$, as we shall see, it has a significant impact on the shape of 
the multiplicity distribution. Moreover, variable $\tilde{m}_{\mathrm{T}}$ is "more similar" to $p_{\mathrm{T}}$ as it vanishes for $p_{\mathrm{T}} \rightarrow 0$ for all particle species, while $m_{\mathrm{T}}$ goes to the species dependent threshold value $m_{\alpha}$. For large transverse momenta both $\tilde{m}_{\mathrm{T}}$ and $m_{\mathrm{T}}$ tend to $p_{\mathrm{T}}$.

Saturation scale $Q_{\mathrm{s}}$ characterizes small Bjorken $x$ gluon cloud developed in the colliding hadrons due to the BFKL-like evolution. It depends upon gluons' $x$ 's which for elastic scattering of massless gluons are given by

$$
x=\frac{p_{\mathrm{T}}}{\sqrt{s}} e^{ \pm y}
$$

where $W=\sqrt{s}$ denotes c.m.s. scattering energy, $p_{\mathrm{T}}$ and $\pm y$ refer to the transverse momentum and to the rapidities of scattered gluons. Saturation scale used originally in Refs. [1, 2] takes the following form

$$
Q_{\mathrm{s}}^{2}=Q_{0}^{2}\left(\frac{x}{x_{0}}\right)^{-\lambda}
$$

where $x_{0}$ is of the order of $10^{-3}-10^{-4}$. Throughout this paper we shall assume $x_{0}=10^{-3}$. Our results, however, are not sensitive neither to this choice nor to the value of $Q_{0}$ for which we take $1 \mathrm{GeV} / c$. It follows from Eq. (4) that in the case of (unidentified) charged particles spectra, scaling variable is naturally given as [4]

$$
\tau_{p_{\mathrm{T}}}=\frac{p_{\mathrm{T}}^{2}}{Q_{\mathrm{s}}^{2}}=\frac{p_{\mathrm{T}}^{2}}{Q_{0}^{2}}\left(\frac{p_{\mathrm{T}}}{W}\right)^{\lambda} .
$$

Due to our choice of $x_{0}$, transverse momentum in Eq. (6) is given in $\mathrm{GeV} / c$ and scattering energy $W$ in $\mathrm{TeV}$. This form of scaling variable has been successfully tested against data for p-p collisions at the LHC [4, 5, 6] and also at lower energies of NA61-SHINE experiment at CERN SPS [15], as well as in the case of heavy ion collisions at RHIC [6].

Here we propose that in the case of identified particles another scaling variable should be used in which $p_{\mathrm{T}}$ is replaced by $\tilde{m}_{\mathrm{T}}\left(\tilde{m}_{\mathrm{T}}-\right.$ scaling $)$, i.e.

$$
\tau_{\tilde{m}_{\mathrm{T}}}=\frac{\tilde{m}_{\mathrm{T}}^{2}}{Q_{0}^{2}}\left(\frac{\tilde{m}_{\mathrm{T}}}{W}\right)^{\lambda} .
$$

This choice is purely phenomenological for the following reasons. Firstly, the gluon cloud is in principle not sensitive to the mass of the particle it finally 
is fragmenting to, so in principle one should take $p_{\mathrm{T}}$ as an argument of the saturation scale. In this case the proper scaling variable would be

$$
\tau_{\tilde{m}_{\mathrm{T}} p_{\mathrm{T}}}=\frac{\tilde{m}_{\mathrm{T}}^{2}}{Q_{0}^{2}}\left(\frac{p_{\mathrm{T}}}{W}\right)^{\lambda} .
$$

We shall show, however, that this choice $\left(\tilde{m}_{\mathrm{T}} p_{\mathrm{T}}-\right.$ scaling $)$ does not really differ numerically from the one given by Eq. (7). On the other hand Eq. (7) has an advantage over (8) since, as we shall see, it allows to calculate analytically many properties of the spectra assuming Tsallis form of the scaling function (1).

Secondly, if we would take seriously kinematics for massive particle production, then Bjorken $x$ would be given by Eq. (4) with $p_{\mathrm{T}}$ replaced by $m_{\mathrm{T}}$. Hence the natural choice for the scaling variable would be $\left(m_{\mathrm{T}}-\right.$ scaling $)$

$$
\tau_{m_{\mathrm{T}}}=\frac{m_{\mathrm{T}}^{2}}{Q_{0}^{2}}\left(\frac{m_{\mathrm{T}}}{W}\right)^{\lambda} .
$$

We shall show, however, that for this choice of the scaling variable GS is not present.

The paper is organized as follows. In Sect. 2 we shall test GS scaling hypothesis on the recent ALICE data for identified particles [20]. We will see that $p_{\mathrm{T}}$ spectra exhibit $\tilde{m}_{\mathrm{T}}$-scaling in variable (7). After that, in Sect. 3 , we shall examine the consequences of GS as far as the energy dependence of total multiplicity and mean transverse momentum is concerned. We shall see that power-like growth of both of them is a natural consequence of GS. Finally in Sect. 4 we shall match hypothesis of GS and phenomenological observation that particle spectra are well described by the Tsallis-like distribution. It will be shown that Tsallis temperature is proportional to the average saturation scale depending only on the scattering energy $W$, whereas Tsallis exponent $n$ is in the first approximation energy independent. Finally, we shall conclude in Sect. 5.

\section{Scaling properties of $\boldsymbol{p}_{\mathrm{T}}$ distributions of identified particles}

Throughout this paper we shall use recent ALICE data for the $p_{\mathrm{T}}$ spectra at $0.9,2.76$ and $7 \mathrm{TeV}$ [20]. In the latter case the data cover wide $p_{\mathrm{T}}$ range from 0.1 to $19 \mathrm{GeV} / c$ (for pions). Unfortunately available pion data for $0.9 \mathrm{TeV}$ span over much narrower range: $0.1-2.5 \mathrm{GeV} / c$, and for $2.76 \mathrm{TeV}$ 
from 2.1 to $19 \mathrm{GeV} / c$, and similarly for kaons and protons where, however, there is no overlap between $0.9 \mathrm{TeV}$ and $2.76 \mathrm{TeV}$ points. For this reason the analysis presented in this paper can be only qualitative.
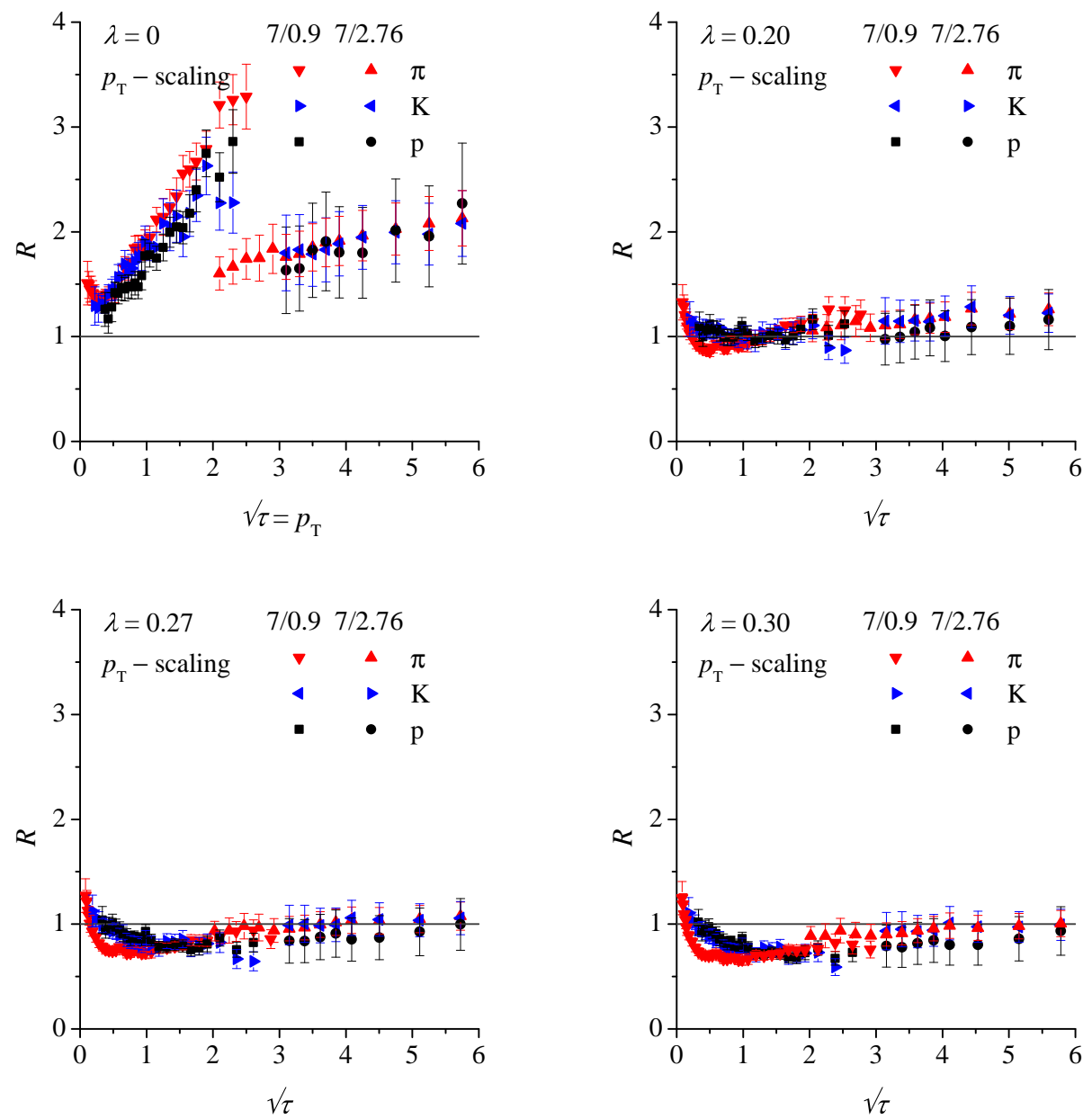

Figure 1: Illustration of geometrical scaling in scaling variable $\tau_{p_{\mathrm{T}}}$. Multiplicity ratios $R_{W_{1} / W_{2}}$ for $W_{1}=7 \mathrm{TeV}$ are plotted as functions of scaling variable $\tau_{p_{\mathrm{T}}}$ for pions (red triangles: "up" for $W_{2}=2.76 \mathrm{TeV}$, "down" for $W_{2}=0.9 \mathrm{TeV}$ ) kaons (blue triangles: "right" for $W_{2}=2.76 \mathrm{TeV}$, "left" for $W_{2}=0.9 \mathrm{TeV}$ ) and protons (back circles for $W_{2}=2.76 \mathrm{TeV}$ and black squares $W_{2}=0.9 \mathrm{TeV}$ ) for different values of the exponent $\lambda$ a) $\lambda=0$, b) $\lambda=0.2$, c) $\lambda=0.27$ and d) $\lambda=0.3$. 
In order to asses the quality of GS we shall apply the method of ratios used previously in Refs. [6, 15] in the context of hadron scattering and in Refs. 3] for DIS. Hypothesis of GS means that particle spectra measured at different energies $W$ are equal when expressed in terms of scaling variable $\tau$ (6) - (8) or (9). Therefore for each particle species $\alpha$ we have

$$
\left.\frac{1}{p_{\mathrm{T}}} \frac{d^{2} N_{\alpha}}{d y d p_{\mathrm{T}}}\right|_{|y|<y_{0}}=\frac{1}{Q_{0}^{2}} F_{\alpha}(\tau)
$$

where $F_{\alpha}(\tau)$ is energy independent function of scaling variable $\tau$ which, however, may depend on particle species $\alpha$. Here $y_{0}$ is the rapidity cut (assumed to be small) which we shall omit in the following. Therefore, if hypothesis of GS is true, we expect that the ratios of multiplicity distributions at two different energies $W_{1}$ and $W_{2}$ (denoted hereafter as $R_{W_{1} / W_{2}}$ ) should be equal to unity if expressed in terms of scaling variable $\tau$. For the purpose of the present analysis we chose $W_{1}=7 \mathrm{TeV}$ as the reference energy.

In Fig. 1 we plot ratios $R_{7 / 0.9}$ and $R_{7 / 2.76}$ as functions of scaling variable $\sqrt{\tau}=\sqrt{\tau_{p_{\mathrm{T}}}}(6)$ for different choices of exponent $\lambda$ entering the definition of the saturation scale $Q_{\mathrm{s}}(5)$. We see that for $\lambda=0$ when $\sqrt{\tau_{p_{\mathrm{T}}}}=p_{\mathrm{T}} / Q_{0}$ ratios are substantially larger than 1 and grow with $p_{\mathrm{T}}$. When $\lambda$ is increased the ratios get smaller and flatter. One can see that the optimal value of exponent $\lambda$ is somewhere between 0.2 and 0.27 . This value is a bit smaller than the value $\lambda=0.27$ obtained in the analysis of unidentified NSD spectra measured by the CMS collaboration at the LHC [4]. We can see that there is a dip in these ratios around $\sqrt{\tau} \sim 1$ which is especially pronounced for pions. Finally, let us remark that we plot $R_{W_{1} / W_{2}}$ only up to $\sqrt{\tau}=6$; we shall see that for larger values of $\sqrt{\tau}$ the ratios get larger than 1 and start growing with $\sqrt{\tau}$. Hence we conclude that there is a window of GS delimited from below by nonperturbative physics and from above by perturbative production of particles with high transverse momentum [15].

Although one can conclude from Fig. 1 that GS works reasonably well for standard scaling variable $\tau_{p_{\mathrm{T}}}(6)$, we are going to examine now the hypothesis that the proper scaling variable for identified particles is $\tau_{\tilde{m}_{\mathrm{T}}}$ of Eq. (7). To this end in Fig. 2 we plot again ratios $R_{7 / 0.9}$ and $R_{7 / 2.76}$ for four different choices of exponent $\lambda$ as functions of scaling variable $\sqrt{\tau_{\tilde{m}_{\mathrm{T}}}}$. We see that the dip for small values of $\tau$ has basically disappeared for kaons and protons and has been largely reduced for pions. Moreover, good quality GS scaling has been achieved for larger value of exponent $\lambda \sim 0.27-0.3$ in fair agreement 
with analysis of DIS [3]. In order to quantify this statement one has to wait, however, until lower energy data is published for larger $p_{\mathrm{T}}$ range similar to the one at $W_{1}=7 \mathrm{TeV}$.
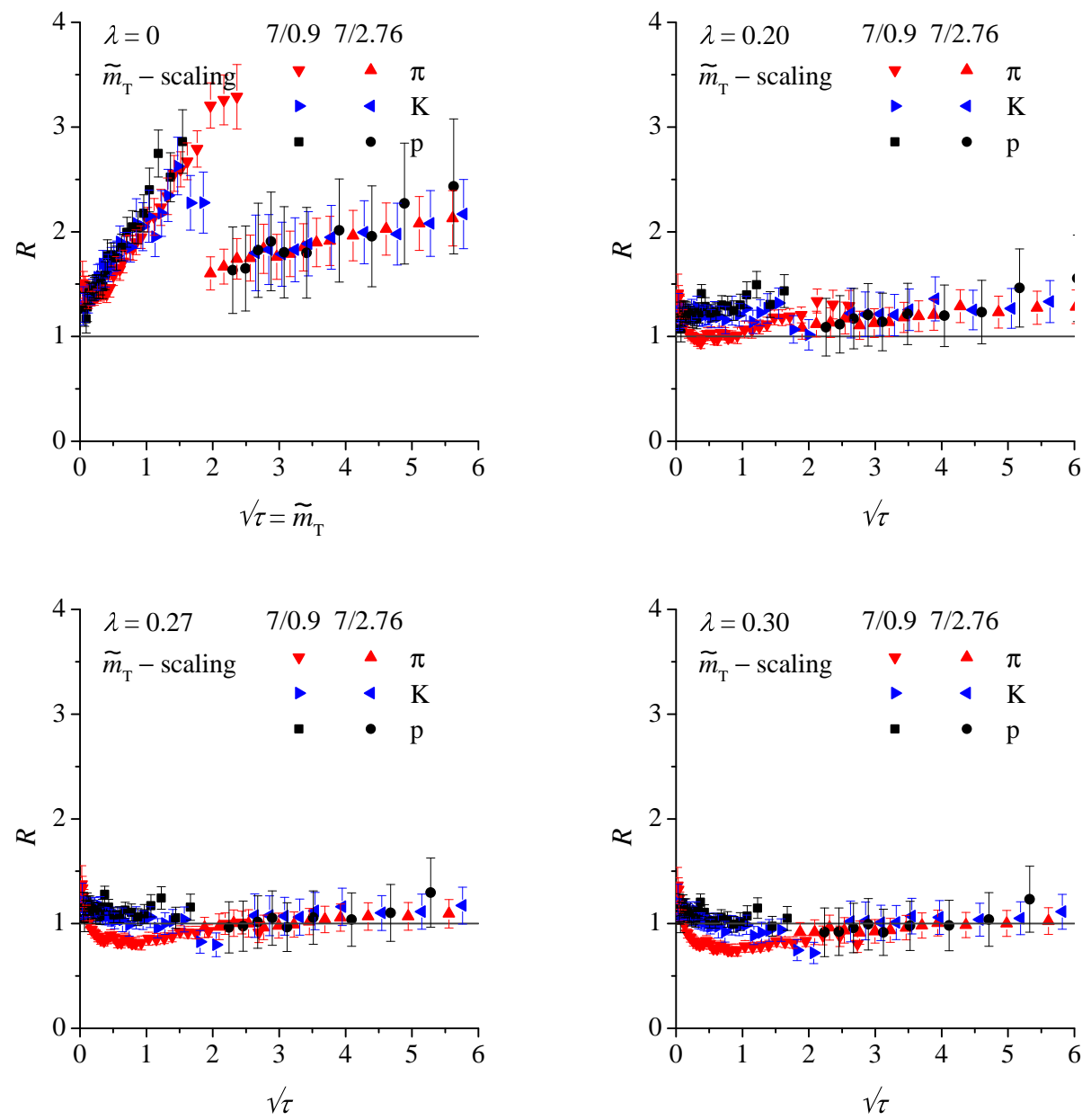

Figure 2: Illustration of geometrical scaling in scaling variable $\tau_{\tilde{m}_{\mathrm{T}}}$. Multiplicity ratios $R_{W_{1} / W_{2}}$ for $W_{1}=7 \mathrm{TeV}$ are plotted as functions of scaling variable $\tau_{m_{\mathrm{T}}}$ for pions (red triangles: "up" for $W_{2}=2.76 \mathrm{TeV}$, "down" for $W_{2}=0.9 \mathrm{TeV}$ ) kaons (blue triangles: "right" for $W_{2}=2.76 \mathrm{TeV}$, "left" for $W_{2}=0.9 \mathrm{TeV}$ ) and protons (back circles for $W_{2}=2.76 \mathrm{TeV}$ and black squares $W_{2}=0.9 \mathrm{TeV}$ ) for different values of the exponent $\lambda$ a) $\lambda=0$, b) $\lambda=0.2$, c) $\lambda=0.27$ and d) $\lambda=0.3$. 
Quantitative analysis should also determine the $p_{\mathrm{T}}$ window where GS should work. Here in Fig. 3 we simply extend the $x$ axis of Fig. 1.c and Fig. 2.c for the case of scaling in variable $\tau_{p_{\mathrm{T}}}$ and $\tau_{\tilde{m}_{\mathrm{T}}}$ respectively. We see, as expected from the properties of $\tilde{m}_{\mathrm{T}}\left(p_{\mathrm{T}}\right)$ as a function of transverse momentum, that the difference between the quality of GS in these two variables shows only for small $\tau$ 's. We can also see from Fig. 3 that GS window closes for $\tau \sim 5$.
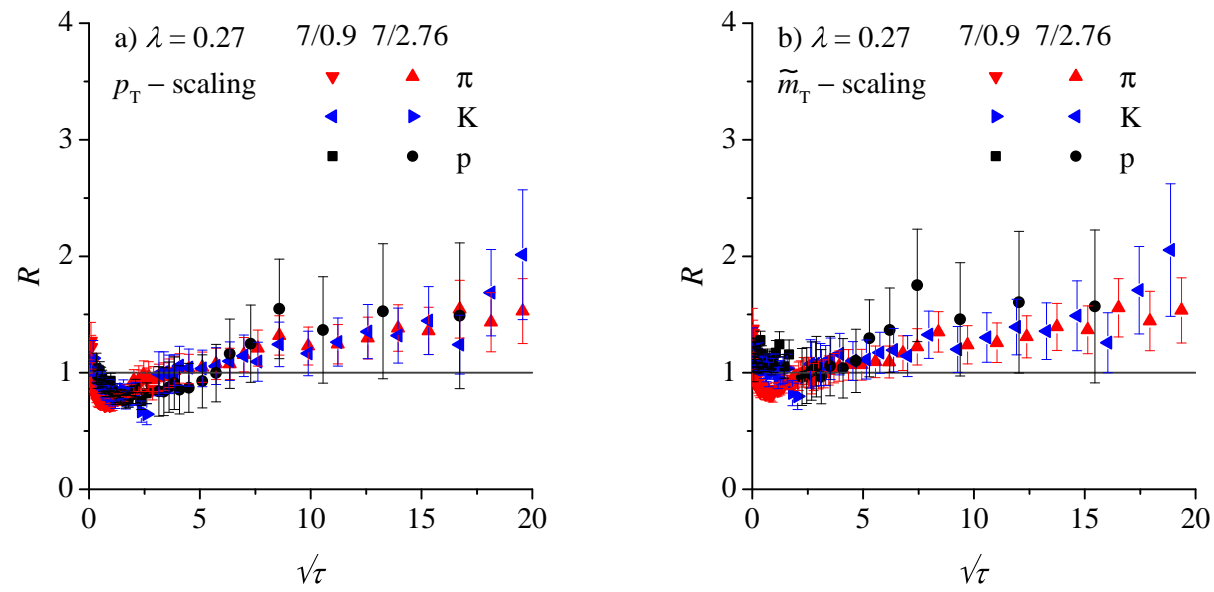

Figure 3: Ratios $R$ from Fig. 1.c and Fig. 2 $\mathrm{c}$ for extended horizontal axis.

Before closing this Section, let us see how scaling properties are affected by going from scaling variable $\tau_{p_{\mathrm{T}}}(6)$ to $\tau_{\tilde{m}_{\mathrm{T}}}$ (7) and what would be the difference in scaling properties if we had chosen $p_{\mathrm{T}}$ as an argument in the saturation scale leading to scaling variable $\tau_{\tilde{m}_{\mathrm{T}} p_{\mathrm{T}}}(8)$, so called $\tilde{m}_{\mathrm{T}} p_{\mathrm{T}}-$ scaling. This is illustrated in Fig. 4.a - 4.c where full symbols refer to the $p_{\mathrm{T}}-$ scaling (6) and open symbols to $\tilde{m}_{\mathrm{T}}-$ scaling or $\tilde{m}_{\mathrm{T}} p_{\mathrm{T}}-$ scaling. One can see very small difference between open symbols indicating that scaling variables $\tau_{\tilde{m}_{\mathrm{T}}}(7)$ and $\tau_{\tilde{m}_{\mathrm{T}} p_{\mathrm{T}}}(8)$ exhibit GS of the same quality. On the contrary $p_{\mathrm{T}}-$ scaling in variable $\tau_{p_{\mathrm{T}}}$ (6) is visibly worse than any form of scaling variable involving $\tilde{m}_{\mathrm{T}}$.

Finally in Fig. 4.d, on the example of protons, we compare $\tilde{m}_{\mathrm{T}}-$ scaling and $m_{\mathrm{T}}$ - scaling for $\lambda=0.27$. One can see that no GS has been achieved in the latter case. Qualitatively the same behavior can be observed for other values of $\lambda$. 

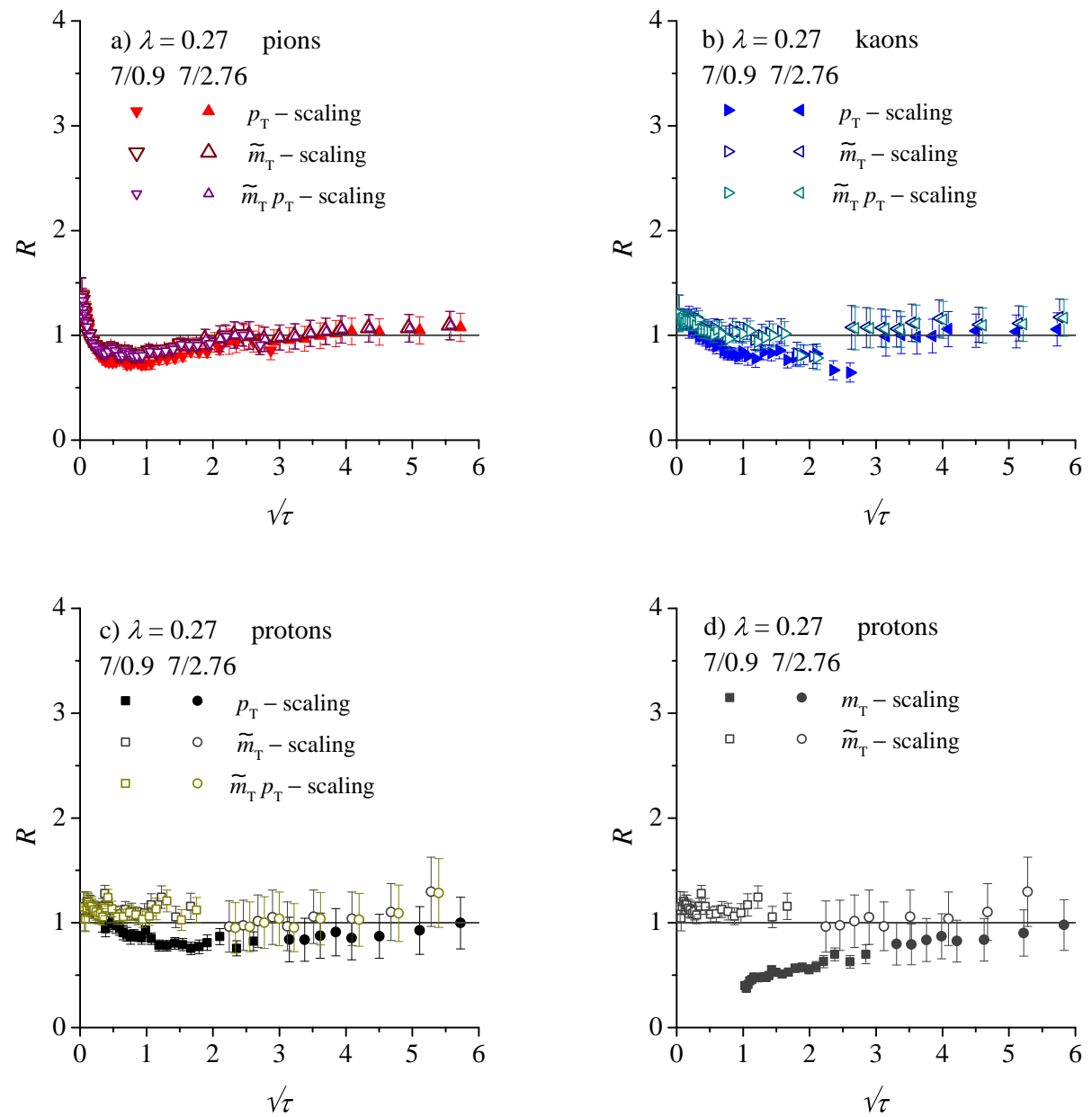

Figure 4: Panels a) - c): comparison of geometrical scaling in three different variables: $\tau_{p_{\mathrm{T}}}$, $\tau_{\tilde{m}_{\mathrm{T}}}$ and $\tau_{\tilde{m}_{\mathrm{T}} p_{\mathrm{T}}}$ for $\lambda=0.27$. Full symbols correspond to ratios $R_{W_{1} / W_{2}}$ plotted in terms of the scaling variable $\tau_{p_{\mathrm{T}}}$, open symbols to $\tau_{\tilde{m}_{\mathrm{T}}}$ and $\tau_{\tilde{m}_{\mathrm{T}} p_{\mathrm{T}}}$, note negligible differences between the latter two forms of scaling variable. Panel a) corresponds to pions, b) to kaons and c) to protons. In panel d) we show comparison of geometrical scaling for protons in scaling variables $\tau_{\tilde{m}_{\mathrm{T}}}$ and $\tau_{m_{\mathrm{T}}}$, no GS can be achieved in the latter case.

Let us remark that recently CMS collaboration has published data on identified spectra [19], however for much smaller range of transverse momenta. Pion and proton spectra have been measured up to $1.5-1.7 \mathrm{GeV} / c$ respectively, whereas kaons up to $1 \mathrm{GeV} / c$ only. In this region ratios $R_{W_{1} / W_{2}}$ 
develop a dip and therefore an attempt to draw conclusions on GS in this case may lead to an underestimate of exponent $\lambda[21$.

The examples presented in this Section illustrate that for identified particles geometrical scaling of good quality is observed for scaling variable (7) within the window $0.5<\sqrt{\tau_{\tilde{m}_{\mathrm{T}}}}<6$ for kaons and protons with lower bound shifted to 1.5 for pions. In the next Section we are going to investigate the consequences of this observation as far as the universal shape of scaling function $F_{\alpha}(\tau)$ is concerned.

\section{Consequences of geometrical scaling}

In what follows we shall assume that scaling variable $\tau=\tau_{\tilde{m}_{\mathrm{T}}}$. We shall also suppress for the moment index $\alpha$ referring to the particle species. Let us first examine the energy dependence of mid rapidity multiplicity density and of average transverse momentum of produced particles. Following Eq. (10), mid rapidity density is given by an integral

$$
\frac{d N}{d y}=\frac{1}{2 Q_{0}^{2}} \int F(\tau) d p_{\mathrm{T}}^{2}
$$

which requires change of variables:

$$
d p_{\mathrm{T}}^{2}=2\left(\tilde{m}_{\mathrm{T}}+m_{\alpha}\right) d \tilde{m}_{\mathrm{T}}
$$

with

$$
d \tilde{m}_{\mathrm{T}}=\frac{Q_{0}}{2+\lambda}\left(\frac{W}{Q_{0}}\right)^{\lambda /(2+\lambda)} \tau^{1 /(2+\lambda)} \frac{d \tau}{\tau} .
$$

Using (13) we arrive at (restoring dependence on particle species $\alpha$ )

$$
\frac{d N_{\alpha}}{d y}=b_{\alpha}\left(\frac{W}{Q_{0}}\right)^{2 \lambda /(2+\lambda)}\left[1+\frac{a_{\alpha}}{b_{\alpha}} \frac{m_{\alpha}}{Q_{0}}\left(\frac{W}{Q_{0}}\right)^{-\lambda /(2+\lambda)}\right] .
$$

We see therefore that mid rapidity identified particle density contains a universal leading term and a correction proportional to the particle mass both rising as powers of energy. The power like rise of mid rapidity density for charged (unidentified) particles has been confirmed up to the LHC energies 22 and the leading power being 0.23 is in agreement with $2 \lambda /(2+\lambda) \approx 0.23$ 
for $\lambda=0.27$ [4] . For large energies and small particle masses one can neglect the second term in Eq. (14).

Constants $a_{\alpha}$ and $b_{\alpha}$ read:

$$
b_{\alpha}=\frac{1}{2+\lambda} \int F_{\alpha}(\tau) \tau^{-\lambda /(2+\lambda)} d \tau, a_{\alpha}=\frac{1}{2+\lambda} \int F_{\alpha}(\tau) \tau^{-(\lambda+1) /(2+\lambda)} d \tau .
$$

We shall show now that GS leads also to the power-like dependence of the mean transverse momentum on the scattering energy. For massive particles we have:

$$
p_{\mathrm{T}}=Q_{0}\left(\frac{W}{Q_{0}}\right)^{\lambda /(2+\lambda)} \tau^{1 /(2+\lambda)} \sqrt{1+2 \frac{m_{\alpha}}{Q_{0}}\left(\frac{W}{Q_{0}}\right)^{-\lambda /(2+\lambda)} \tau^{-1 /(2+\lambda)}} .
$$

We see that for large energies the second term under the square root is suppressed (and also for small masses) so after expansion for large $W$ we obtain

$$
p_{\mathrm{T}}=Q_{0}\left(\frac{W}{Q_{0}}\right)^{\lambda /(2+\lambda)} \tau^{1 /(2+\lambda)}+m_{\alpha}+\ldots
$$

We define mean transverse momentum as:

$$
\left\langle p_{\mathrm{T}}\right\rangle=\frac{\frac{1}{2 Q_{0}^{2}} \int p_{\mathrm{T}} F_{\alpha}(\tau) d p_{\mathrm{T}}^{2}}{\frac{1}{2 Q_{0}^{2}} \int F_{\alpha}(\tau) d p_{\mathrm{T}}^{2}}
$$

Denominator of (18) is given by Eq. (14) whereas the numerator, after expanding in powers of $m_{\alpha}$ reads

$$
\frac{\mathrm{num} .}{Q_{0}}=\left(\frac{W}{Q_{0}}\right)^{2 \lambda /(2+\lambda)}\left(c_{\alpha}\left(\frac{W}{Q_{0}}\right)^{\lambda /(2+\lambda)}+2 b_{\alpha} \frac{m_{\alpha}}{Q_{0}}\right)
$$

with

$$
c_{a}=\frac{1}{2+\lambda} \int F_{\alpha}(\tau) \tau^{-(\lambda-1) /(2+\lambda)} d \tau
$$

Hence mean $p_{\mathrm{T}}$ reads

$$
\left\langle p_{\mathrm{T}}\right\rangle=Q_{0} \frac{c_{\alpha}\left(\frac{W}{Q_{0}}\right)^{\lambda /(2+\lambda)}+2 b_{\alpha} \frac{m_{\alpha}}{Q_{0}}}{b_{\alpha}+a_{\alpha} \frac{m_{\alpha}}{Q_{0}}\left(\frac{W}{Q_{0}}\right)^{-\lambda /(2+\lambda)}} \simeq Q_{0} \frac{c_{\alpha}}{b_{\alpha}}\left(\frac{W}{Q_{0}}\right)^{\lambda /(2+\lambda)}+m_{\alpha}\left(2-\frac{a_{\alpha} c_{\alpha}}{b_{\alpha}^{2}}\right) .
$$


We see that mean transverse momentum behaves as a constant (proportional to the particle mass) plus a power of energy, which is also confirmed by the recent data up to the LHC energies [22]. Let us remark that formulae (14) and 20) imply in the leading order

$$
\left\langle p_{\mathrm{T}}\right\rangle=A+B \sqrt{d N / d y}
$$

\section{Universal shape of geometrical scaling and Tsallis-like parametri- zation}

We shall now be more specific and use a particular form of function $F_{\alpha}(\tau)$. This will allow us to calculate explicitly constants $a_{\alpha}, b_{\beta}$ and $c_{\alpha}$. To this end we shall use the experimental observation that identified particles spectra can be well described in terms of Tsallis-like parametrization of Eq. (1) with species dependent temperature $T=T_{\alpha}$ and exponent $n=n_{\alpha}$. In actual fits to the data $n_{\alpha}$ is of the order 5 to 9 [19], therefore we may use an approximation

$$
C_{\alpha} \simeq \frac{\gamma_{\alpha}}{T_{\alpha}^{2}}
$$

where constant $\gamma_{\alpha}$ restores the correct normalization being only a function of $n_{\alpha}$. Inserting (22) and (13) into (1) we obtain:

$$
\frac{d^{2} N_{\alpha}}{d y d p_{\mathrm{T}}^{2}}=\frac{\gamma_{\alpha}}{2 T_{\alpha}^{2}} \frac{d N_{\alpha}}{d y}\left[1+\frac{Q_{0}\left(\frac{W}{Q_{0}}\right)^{\lambda /(2+\lambda)} \tau^{1 /(2+\lambda)}}{n_{\alpha} T_{\alpha}}\right]^{-n_{\alpha}}
$$

Finally, we shall use leading term for energy dependence of the mid rapidity multiplicity distribution (14) which gives

$$
\frac{d^{2} N_{\alpha}}{d y d p_{\mathrm{T}}^{2}}=\frac{\gamma_{\alpha}}{2 T_{\alpha}^{2}} b_{\alpha}\left(\frac{W}{Q_{0}}\right)^{2 \lambda /(2+\lambda)}\left[1+\frac{Q_{0}\left(\frac{W}{Q_{0}}\right)^{\lambda /(2+\lambda)} \tau^{1 /(2+\lambda)}}{n_{\alpha} T_{\alpha}}\right]^{-n_{\alpha}}
$$

The right hand side of Eq. 24 should be an energy independent function

of scaling variable $\tau$ only. Within approximations used so far there exists a simple solution to this requirement:

$$
T_{\alpha}=\kappa_{\alpha} Q_{0}\left(\frac{W}{Q_{0}}\right)^{\lambda /(2+\lambda)}
$$


where $\kappa_{\alpha}$ is a constant. Therefore GS predictions for Tsallis parameters are that $T_{\alpha}$ depends on energy as a power ${ }^{1}$, whereas $n_{\alpha}$ is a constant. A complete fit to high energy data for charged (unidentified) particles from NA49 energies up to the LHC [23] shows rather small variation (of the order of $10 \%$ ) of parameter $q(W)$ related to $n$ from Eq. (1) in the following way:

$$
n(W)=\frac{1}{q(W)-1}
$$

which, however, translates into rather strong energy dependence of $n(W)$, especially for smaller energies where $q(W)$ is only slightly bigger than 1 . Similar conclusion - as far as the energy dependence of the Tsallis parameters for identified particles is concerned - has been found in Ref. 24] with temperature hardly depending on energy. One should note, however, that the multiplicity distribution used in Ref. [24] slightly differs from the one of Eq. (1).

The solution with constant $n_{\alpha}$ has a number of corrections which in the present approach can be studied in a systematic way. Ignoring them for them the moment we arrive at the universal scaling function which takes the following form:

$$
F_{\alpha}(\tau)=\frac{\gamma_{\alpha} b_{\alpha}}{2 \kappa_{\alpha}^{2}}\left[1+\frac{\tau^{1 /(2+\lambda)}}{n_{\alpha} \kappa_{\alpha}}\right]^{-n_{\alpha}} .
$$

The solution for $T_{\alpha}$ given by Eq. 25) can be interpreted in terms of the saturation scale, $Q_{\mathrm{s}}(5)$ which for $\tilde{m}_{\mathrm{T}}$-scaling takes the following form:

$$
Q_{\mathrm{s}}\left(\tilde{m}_{\mathrm{T}}\right)=Q_{0}\left(\frac{\tilde{m}_{\mathrm{T}}}{W}\right)^{-\lambda / 2} .
$$

For quantities integrated over transverse momentum one introduces another saturation scale, $\bar{Q}_{\mathrm{s}}$ which has a meaning of an average transverse momentum, or in this case average value of $\tilde{m}_{\mathrm{T}}$, and can be thought of as a solution of an equation [25]:

$$
\bar{Q}_{\mathrm{s}}=Q_{\mathrm{s}}\left(\bar{Q}_{\mathrm{s}}\right)
$$

which gives

$$
\bar{Q}_{\mathrm{s}}=Q_{0}\left(\frac{W}{Q_{0}}\right)^{\lambda /(2+\lambda)}
$$

\footnotetext{
${ }^{1}$ This dependence is, however, rather weak for large energies.
} 
We see therefore that parameter $T_{\alpha}$, Tsallis temperature 25$)$, is proportional to the average saturation scale $\bar{Q}_{\mathrm{s}}$ with proportionality constant $\kappa_{\alpha}$ which depends on particle species $\alpha$. Constants $\kappa_{\alpha}$ have been fitted to thermal distributions in Ref. [26] and they are of the order of 0.1. Similar solution for the unidentified spectra has been discussed recently in Ref. [27].

Constants $a_{\alpha}, b_{\alpha}$ and $c_{\alpha}$ can be calculated analytically for $F_{\alpha}(\tau)$ given by Eq. (27):

$$
\begin{aligned}
a_{\alpha} & =\frac{\gamma_{\alpha} b_{\alpha}}{2 \kappa_{\alpha}^{2}}\left(n_{\alpha} \kappa_{\alpha}\right) B\left(1, n_{\alpha}-1\right), \\
b_{\alpha} & =\frac{\gamma_{\alpha} b_{\alpha}}{2 \kappa_{\alpha}^{2}}\left(n_{\alpha} \kappa_{\alpha}\right)^{2} B\left(2, n_{\alpha}-2\right), \\
c_{\alpha} & =\frac{\gamma_{\alpha} b_{\alpha}}{2 \kappa_{\alpha}^{2}}\left(n_{\alpha} \kappa_{\alpha}\right)^{3} B\left(3, n_{\alpha}-3\right)
\end{aligned}
$$

where $B(x, y)$ is Euler beta function. The second equation should be understood as a normalization condition for $\gamma_{\alpha}$ :

$$
\gamma_{\alpha}=\frac{2}{n_{\alpha}^{2} B\left(2, n_{\alpha}-2\right)}
$$

which is independent of $\kappa_{\alpha}$, as expected. With this normalization we arrive at:

$$
a_{\alpha}=\frac{b_{\alpha}}{\kappa_{\alpha}} \frac{n_{\alpha}-2}{n_{\alpha}}, \quad c_{\alpha}=b_{\alpha} \kappa_{\alpha} \frac{2 n_{\alpha}}{n_{\alpha}-3}
$$

with $b_{\alpha}$ being a free constant which can be fitted from the energy dependence of the mid rapidity density (14). The coefficient governing the constant piece in a formula for $\left\langle p_{\mathrm{T}}\right\rangle$ (20) is given by:

$$
2-\frac{a_{\alpha} c_{\alpha}}{b_{\alpha}^{2}}=-\frac{2}{n_{\alpha}-3} .
$$

It is important to note that this coefficient is negative for values of $n_{\alpha}$ extracted from the data [19] and that the constant piece in Eq. 200 is growing with particle mass. Note, however, that there might exist a nonperturbative contribution to this term which is beyond control in the present approach.

\section{Conclusions}

In this paper we have demonstrated using recent ALICE pp data for identified particles at three LHC energies [20] that transverse momentum 
spectra exhibit geometrical scaling in variable $\tau_{\tilde{m}_{\mathrm{T}}}=\left(\tilde{m}_{\mathrm{T}} / Q_{0}\right)^{2}\left(\tilde{m}_{\mathrm{T}} / W\right)^{\lambda}$. It is impossible at present to asses quantitatively the quality of this scaling, since the data for $W=0.9$ and $2.76 \mathrm{TeV}$ published so far, do not overlap (or have very small overlap) in $p_{\mathrm{T}}$. It can be, however, seen "by eye" that the $\tilde{m}_{\mathrm{T}^{-}}$ scaling works better for identified particles than the "standard" $p_{\mathrm{T}}$-scaling. Moreover, the optimal value of the exponent $\lambda$ is definitely closer to the DIS value of 0.32 than in the case of the $p_{\mathrm{T}^{-}}$scaling for (unidentified) charged particles where it is equal to 0.27 [4]. This statements can be quantified using the method of ratios [3] once data in the full $p_{\mathrm{T}}$ range is published.

One of the immediate consequences of geometrical scaling in variable $\tau_{\tilde{m}_{T}}$ is power-like growth of multiplicity and average transverse momentum with scattering energy $W$. We have shown in Refs. 44 that the values of the pertinent exponents for (unidentified) charge particles are in agreement with experimental fits. In the present work we have shown that the coefficients of this growth and possible constant terms are calculable in terms of the universal scaling function $F(\tau)$ (15) 19). From Eqs. (14) and (20) one can in principle determine constants $a_{\alpha}, b_{\alpha}$ and $c_{\alpha}$ once the pertinent data is available.

We have also made an attempt to predict constants $a_{\alpha}, b_{\alpha}$ and $c_{\alpha}$ assuming certain form of the scaling function $F(\tau)$. To this end we have used an experimental observation that identified particle spectra for small and intermediate values of $p_{\mathrm{T}}$ are well described by the Tsallis-like parametrization (1). This allowed us to relate Tsallis temperature to the energy dependent average saturation scale $\bar{Q}_{\mathrm{s}}(29)$. Within approximation used in this paper Tsallis exponent $n$ remains energy independent. Corrections to this solution are in principle calculable in the present approach.

Phenomenological findings of the present paper call for deeper theoretical understanding. The meaning of constant $\kappa_{\alpha}$ in Eq. 25) and species dependence of exponent $n_{\alpha}$ are the most obvious examples. This may, however, require to construct a nonperturbative fragmentation model which is beyond the scope of the present paper.

\section{Acknowledgements}

The author would like to thank Larry McLerran for discussion and remarks and the ALICE Collaboration for an access to the data on the $p_{\mathrm{T}}$ spectra. This research has been supported by the Polish NCN grant 2011/01/B/ ST2/00492. 


\section{References}

[1] A. M. Stasto, K. J. Golec-Biernat, and J. Kwiecinski, Phys. Rev. Lett. 86, 596 (2001) hep-ph/0007192.

[2] K. J. Golec-Biernat, and M. Wüsthoff, Phys. Rev. D 59, 014017 (1998) [hep-ph/9807513], and Phys. Rev. D 60, 114023 (1999) hepph/9903358.

[3] M. Praszalowicz and T. Stebel, JHEP 1303, 090 (2013) arXiv:1211.5305 [hep-ph]], and JHEP 1304, 0169 (2013) arXiv:1302.4227 [hep-ph].

[4] L. McLerran, and M. Praszalowicz, Acta Phys. Pol. B 41, 1917 (2010) arXiv:1006.4293 [hep-ph]], and Acta Phys. Pol. B 42, 99 (2011), arXiv:1011.3403 [hep-ph]].

[5] M. Praszalowicz, Phys. Rev. Lett. 106, 142002 (2011), arXiv:1101.0585 [hep-ph]].

[6] M. Praszalowicz, Acta Phys. Pol. B 42, 1557 (2011) arXiv:1104.1777 [hep-ph]], and in Proceedings of the 47th Rencontres de Moriond, La Thuile, 2012, p. 265 arXiv:1205.4538 [hep-ph]].

[7] L. V. Gribov, E. M. Levin, and M. G. Ryskin, Phys. Rept. 100, 1 (1983);

[8] A. H. Mueller, and J-W. Qiu, Nucl. Phys. 268, 427 (1986); A. H. Mueller, Nucl. Phys. B558, 285 (1999).

[9] A. H. Mueller, Parton Saturation: An Overview, arXiv:hep-ph/0111244.

[10] L. McLerran, Acta Phys. Pol. B 41, 2799 (2010) arXiv:1011.3203 [hep$\mathrm{ph}]$.

[11] J. Jalilian-Marian, A. Kovner, A. Leonidov, and H. Weigert, Nucl. Phys. B504, 415 (1997), and Phys. Rev. D59, 014014 (1998);

E. Iancu, A. Leonidov, and L. D. McLerran, Nucl. Phys. A692, 583 (2001);

E. Ferreiro, E. Iancu, A. Leonidov, and L. D. McLerran, Nucl. Phys. A703, 489 (2002). 
[12] I. Balitsky, Nucl. Phys. B463, 99 (1996);

Y. V. Kovchegov, Phys. Rev. D60, 034008 (1999), and Phys. Rev. D61, 074018 (2000).

[13] S. Munier, and R. B. Peschanski, Phys. Rev. Lett. 91, 232001 (2003) [hep-ph/0309177], and Phys. Rev. D 69, 034008 (2004) hep$\mathrm{ph} / 0310357$.

[14] L. D. McLerran, and R. Venugopalan, Phys. Rev. D49, 2233 (1994), Phys. Rev. D49, 3352 (1994), and Phys. Rev. D50, 2225 (1994).

[15] M. Praszalowicz, Phys. Rev. D 87, 071502(R) (2013) arXiv:1301.4647 [hep-ph]], and arXiv:1304.1867 [hep-ph].

[16] T. Stebel, arXiv:1305.2583 [hep-ph].

[17] R Hagedorn, Nuovo Cim. Suppl.3, 147 (1965).

[18] C. Tsallis, J. Stat. Phys. 52479 (1988);

T. S. Biró, G. Purcsel, and K. Ürmössy, Eur. Phys. J. A 40, 325 (2009).

[19] S. Chatrchyan et al. [CMS Collaboration], Eur. Phys. J. C 72, 2164 (2012) [arXiv:1207.4724 [hep-ex]].

[20] K. Aamodt et al. [ALICE Collaboration], Eur. Phys. J. C 71, 1655 (2011) [arXiv:1101.4110 [hep-ex]];

A. Ortiz Velasquez [ALICE Collaboration], Nucl. Phys. A904-905 2013, 763c (2013) arXiv:1210.6995 [hep-ex]] (ALICE preliminary).

[21] M. Praszalowicz, unpublished;

F. Sikler, private communication.

[22] K. Aamodt et al. [ALICE Collaboration], Eur. Phys. J. C 65, 111 (2010) arXiv:0911.5430 [hep-ex]], and Eur. Phys. J. C 68, 89 (2010) arXiv:1004.3034 [hep-ex], and Eur. Phys. J. C 68, 345 (2010) arXiv:1004.3514 [hep-ex].

[23] M. Rybczynski, Z. Wlodarczyk and G. Wilk, J. Phys. G 39, 095004 (2012) arXiv:1203.6787 [hep-ph]].

[24] J. Cleymans, G. I. Lykasov, A. S. Parvan, A. S. Sorin, O. V. Teryaev and D. Worku, Phys. Lett. B 723, 351 (2013) arXiv:1302.1970 [hep-ph]]. 
[25] D. Kharzeev, E. Levin and M. Nardi, Nucl. Phys. A 747, 609 (2005) hep-ph/0408050.

[26] L. McLerran, M. Praszalowicz and B. Schenke, arXiv:1306.2350 [hep$\mathrm{ph}$.

[27] M. Rybczynski, Z. Wlodarczyk and G. Wilk, Acta Phys. Polon. Supp. 6, 507 (2013) [arXiv:1212.1281]. 